\section{The size of focus samples in multiple-category concept identification*:}

\author{
MARK B. REEVE, PETER G. POLSON, and JACK L. DUNHAM \\ The University of Texas at Austin, Austin, Tex. 78712
}

Introductory psychology students solved unidimensional four-category concept identification $(\mathrm{CI})$ problems. In the experimental group $(\mathrm{N}=38)$ two dimensions were redundant and relevant, while two control groups $(N=18$ in each) solved problems where only one of these dimensions was relevant. Of the Ss in the experimental group, $26.3 \%$ were assessed as solving the problem using both relevant dimensions. To account for the data, a dimension selection theory of multiple-category $\mathrm{CI}$ is described, which incorporates the assumption that $S$ is attending to more than a single dimension and employing a focusing strategy.

Recent theories of concept identification (CI) (Bower \& Trabasso, 1964; Restle, 1962; Trabasso \& Bower. 1968) have mainly dealt with two-category CI problems in which the correct classification of a stinulus depends upon the values of a single binary-valued dimension. The two-category CI problem is in some respects a special case within the general CI paradigm. The correct classification depends only upon the values of a single binary dimension. (iven a knowledge of the problem's structure, $S$ can construct a complete response rule from information about the correct classification of the current instance. In Cl problems where the number of categories (n) is greater than two, $S$ needs information from more than a single trial in order to construct a complete response rule.

In view of the empirical support for these theorics of iwo-category $\mathrm{Cl}$, it would seem fruitful to extend them to the more complex $\mathrm{Cl}$ tasks. Bower \& Trabasso (1964) did propose a model for four-category $\mathrm{Cl}$ tasks. They assumed that the processes involved in CI where $n$ is greater than two were essentially the same as those used when n equals two. However, major modifications seem necessary to allow for the differences in the amount of information available to $\mathrm{S}$ on a single trial Any single trial provides $S$ information only about the response to the value of the dimension on the current stimulus. It must be assumed that $S$ in some way constructs a partial hypothesis about the solution of the problem on the basis of available information. Later, as he receives more information, the partial rule can be completed. The model outlined in the

*This work was performed at The University of Texas at Auslin while the first author was supported by a P'ublic Health Service predoctoral fellowship, $1101 \mathrm{MH} 45813-01$, from tho National Institute of Mental Health, Public Health Service. present study describes $\mathrm{S}$ as constructing hypotheses by selecting a dimension and associating feedback to the values of that dimension. Hence, the model will be referred to as the hypothesis construction (HC) model.

The HC model assumes four processes: dimension selection, feedback association, response production, and evaluation. In this model, when $S$ constructs hypotheses, he attends to a single dimension. On any trial, if $S$ has a response conditioned to the value of the attended-to dimension, he gives that response. An error of comission occurs if this response is wrong. In this event, $S$ rejects his hypothesis and resamples with replacement. The probability of sampling any dimension is a function of the salience of that dimension. On any trial. if $S$ does not have a response cunditioned to the value of the attended-to dimension, he guesses with each of the $n$ responses having an equal probability of occurring. trrors due to incorrect guesses do not lead to resampling. When presented with the fecdhack on a gucssing trial, $S$ conditions that feedback to the unconditioned value with probability $\theta$. When $S$ is attending to the relevant dimension. he will never make an error of commission and will eventually learn responses to all $n$ values of that dimension. When $S$ is attending to an irrelcvant dimension, he will condition responses to one or more values of that dimension, eventually make an error of commission, and resample.

Trabasso \& Bower (1968) have recently proposed a major modification of their 1964 theory. The crucial difference is that the new model assumes that $S$ can attend to more than one dimension at a time. To eliminate irrelevant dimensions from his sample, S would employ a focusing strategy, rejecting those dimensions on a success trial which would have led to an incorrect response. To test this theory, a study was conducted by Trabasso \& Bower
(1968) using an experimental problem with two dimensions redundant and relevant. The results of this study show that for two-category CI problems. some Ss solve the problem on the basis of both relevant dimensions. This finding has been interpreted as supporting a focusing model which asserts that $S$ may attend to more than one dimension at a time.

The purpose of the present study was to examine the necessity of similar attentional and focusing assumptions in a dimension selection theory for CI problems with $n$ greater than two. As in the Trabasso \& Bower (1968) study, if a significant proportion of the $\mathrm{Ss}$ demonstrate a two-dimension solution for a problem with two dimensions redundant and relevant, the assumption that $S$ attends to a single dimension while solving multiple-category problems is false.

\section{SUBJECTS}

The Ss were 74 introductory psychology students at The University of Texas at Austin. They were assigned randomly to one of three groups with the restriction that approximately half were assigned to the experimental group and the remainder were divided into two control groups.

\section{DESIGN AND PROCEDURE}

All Ss were well instructed as to the nature of the task, the dimensions and their values. and the possible responses. To familiarize them with the procedure and the apparatus, all $S s$ were required to solve a practice problem with a single relevant and a single irrelevant dimension.

Following the practice problem, Ss solved a four category unidimensional $\mathrm{CI}$ problem presented by the anticipation method. The stimuli varied along four dimensions (color, shape, number of figures, and position of a black dot) with four values per dimension. The responses were the letters A, B, C, and D. The Ss in the experimental group solved a problem in which number and shape were redundant and relevant. One control group solved a problem in which shape was the only relevant dimension, and the other control group had number as the only relevant dimension

After reaching criterion of 16 consecutive correct responses, each $S$ completed a printed questionnaire to assess whether he had solved the problem on the basis of one or both of the relevant dimensions. This questionnaire was similar to that used by Trabasso \& Bower (1968, Chap. 3) but consisted of 17 questions. Each of the first 16 questions asked about a particular value of a particular dimension. The $S$ was instructed to circle an $A, B, C$, or $\mathrm{D}$, according to the category in which a card with that value would belong. If $S$ did not know or could not decide the correct 
response, he was instructed to circle an $\mathrm{X}$. The last question required $S$ to describe the system he used to sort the cards.

\section{RESULTS}

All Ss could readily be classified as to the dimension or dimensions on which they had based their solution. Of the $38 \mathrm{Ss}$ in the experimental group, 10 were assessed as having solved the problem using both relevant cues. The remaining Ss solved on the basis of a single cue.

Summary statistics for the trial of last error and the total errors are given in Table 1. There was no significant difference among the three groups for trial of last error, $F(2,71)<1.0$, or for total errors, $\mathrm{F}(2,71)<1.0$.

\section{DISCUSSION}

Of the $S s$ in the experimental group, $26.3 \%$ solved the problem using both relevant dimensions. These findings necessitate a model of multiple-category CI which allows for $S$ attending to more than a single aspect of the stimulus. An $\mathrm{HC}$ model could account for the data by the addition of attentional and focusing assumptions similar to those made by Trabasso \& Bower (1968). A tentative version of an $\mathrm{HC}$ model with this extension makes the following assumptions about the learning of unidimensional multiple-category $\mathrm{CI}$ problems.

On the initial trial and on any other trial where he resamples, $S$ selects from the set of all dimensions a subset of dimensions to which he attends. As in the Trabasso \& Bower (1968) theory, the selection mechanism is assumed to be a random sampling process in which the probability of sampling any dimension is a function of the salience of the dimension.

All values of the sampled dimension are assumed to be unconditioned immediately following sampling. On the trial in which sampling occurred or on any other trial, the feedback is conditioned with probability $\theta$ to all current unconditioned values of the attended-to dimensions.

Trabasso \& Bower (1968, Chap. 2) assume that $S$ alternates between a search mode and a test mode of operation. In the search mode, $S$ selects a set of hypotheses consistent with current feedback. The $S$ then shifts to a test mode for the next stimulus in the series. In the multiple-category problem, this distinction cannot be sharply defined. Rather than

Table 1

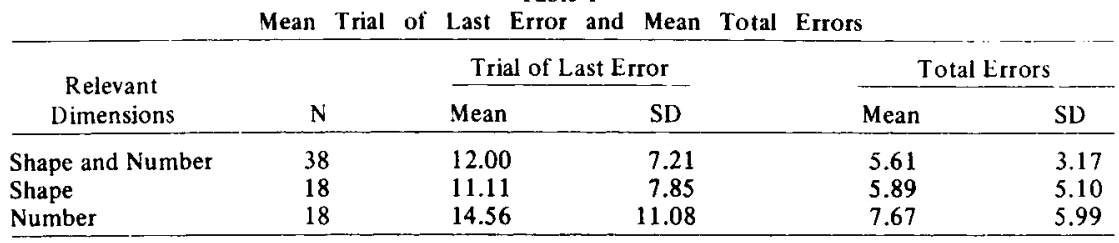

sampling hypotheses, $\mathrm{S}$ samples dimensions and then constructs hypotheses consistent with the feedback given with each instance. He may have the opportunity to test the partial hypothesis prior to completely specifying it.

On any trial, $S$ is confronted with a complex and possibly conflicting stimulus pattern to which to respond. The value of each of the sampled dimensions may be conditioned to any of the $\mathrm{n}$ responses, or it may be unconditioned. Consistent with Trabasso \& Bower (1968), the response rule to be assumed is as follows: When presented with a situation where all values are conditioned to a common response, $S$ makes that response. In any other situation, one value is picked at random, and $S$ makes the response associated with the sampled value. If that value is unconditioned, $S$ guesses with each of the $n$ responses having equal probability of occurring.

The final process is for eliminating dimensions from the focus sample. Again following Trabasso \& Bower (1968), it is assumed that an error of commission leads $S$ to resample. When $S$ is responding for a reason and is correct, those dimensions whose values were associated with incorrect responses are eliminated from the sample. Trabasso and Bower did not have to deal with the third possible outcome, where $S$ guesses. It is assumed that regardless of whether or not he guesses correctly, the action is the same as when $S$ is correct for a reason. Those dimensions leading to an error of commission are eliminated from the sample.

The HC model with the attentional and focusing assumptions can be briefly summarized as follows: (1) The S's current state is characterized by his current sample, and the response (if any) associated to each value of each dimension on his sample. (2) The $S$ changes state by associating responses to values previously unconditioned, eliminating dimensions from the sample, or resampling. (3) When one or more unconditioned values are presented with feedback, that feedback is conditioned to these values with probability $\theta$. (4) When $S$ makes an error of commission, he resamples. A sample of dimensions is chosen, with replacement, from the set of all dimensions. The probability of choosing any dimension is a function of its salience. (5) When $S$ makes a correct response or is guessing, dimensions having values conditioned to an incorrect response are dropped from the sample. (6) On any trial, the probability of each response is proportional to the number of values conditioned to that response. Any unconditioned value is assumed to contribute equally to the probability of each $n$ responses.

In essence, the extended $\mathrm{HC}$ model requires that $S$ hold and manipulate simultaneously two or more partial hypotheses of the type described in the introduction. This seems unlikely in light of what is known about the memory and processing constraints of human Ss. It may be necessary to consider theories where $S$ is seen as processing information in a quite different manner. For instance, it is logically possible to use information from a previous trial in conjunction with the information of the current trial to isolate the relevant dimension. In any event, it is clear that any theory of multiple-category CI must take into consideration that a significant proportion of Ss, given a problem with two dimensions redundant and relevant, do solve with both dimensions.

\section{REFERENCES}

BOWER, G. H., \& TRABASSO, T. Concept identification. In R. C. A tkinson (Ed.), Studies in mathematical psychology. Stanford: Stanford University Press, 1964. Pp. 32-94.

RESTLE, F. The selection of strategies in cue learning. Psychological Review, 1962, 69. 11-19.

TRABASSO, T., \& BOWER, G. H. Attention in learning. New York: Wiley, 1968. 